NASA/CR-1999-209688

ICASE Report No. 99-39

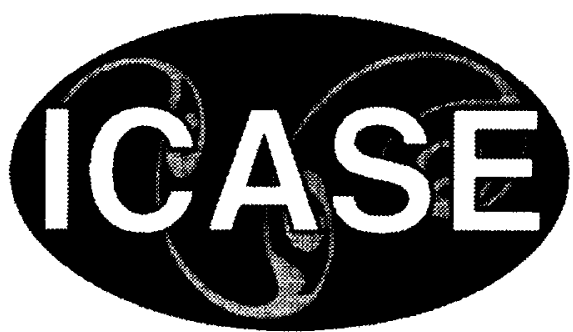

\title{
Characterization of Sound Radiation by Unresolved Scales of Motion in Computational Aeroacoustics
}

Robert Rubinstein and Ye Zhou

ICASE, Hampton, Virginia 


\section{The NASA STI Program Office ... in Profile}

Since its founding, NASA has been dedicated to the advancement of aeronautics and space science. The NASA Scientific and Technical Information (STI) Program Office plays a key part in helping NASA maintain this important role.

The NASA STI Program Office is operated by Langley Research Center, the lead center for NASA's scientific and technical information. The NASA STI Program Office provides access to the NASA STI Database, the largest collection of aeronautical and space science STI in the world. The Program Office is also NASA's institutional mechanism for disseminating the results of its research and development activities. These results are published by NASA in the NASA STI Report Series, which includes the following report types:

- TECHNICAL PUBLICATION. Reports of completed research or a major significant phase of research that present the results of NASA programs and include extensive data or theoretical analysis. Includes compilations of significant scientific and technical data and information deemed to be of continuing reference value. NASA counter-part or peer-reviewed formal professional papers, but having less stringent limitations on manuscript length and extent of graphic presentations.

- TECHNICAL MEMORANDUM. Scientific and technical findings that are preliminary or of specialized interest, e.g.. quick release reports, working papers, and bibliographies that contain minimal annotation. Does not contain extensive analysis.

- CONTRACTOR REPORT. Scientific and technical findings by NASA-sponsored contractors and grantees.
- CONFERENCE PUBLICATIONS. Collected papers from scientific and technical conferences, symposia, seminars, or other meetings sponsored or co-sponsored by NASA.

- SPECIAL PUBLICATION. Scientific, technical, or historical information from NASA programs, projects, and missions, often concerned with subjects having substantial public interest.

- TECHNICAL TRANSLATION. Englishlanguage translations of foreign scientific and technical material pertinent to NASA's mission.

Specialized services that help round out the STI Program Office's diverse offerings include creating custom thesauri, building customized databases, organizing and publishing research results ... even providing videos.

For more information about the NASA STI Program Office, you can:

- Access the NASA STI Program Home Page at http://www.sti.nasa.gov/STIhomepage.html

- Email your question via the Internet to help@sti.nasa.gov

- Fax your question to the NASA Access Help Desk at (301) 621-0134

- Phone the NASA Access Help Desk at (301) 621-0390

- Write to: NASA Access Help Desk NASA Center for AeroSpace Information 7121 Standard Drive Hanover, MD 21076-1320 
NASA/CR-1999-209688

ICASE Report No. 99-39

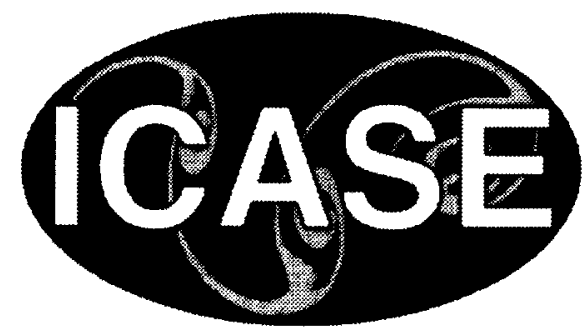

\section{Characterization of Sound Radiation by Unresolved Scales of Motion in Computational Aeroacoustics}

Robert Rubinstein and Ye Zhou

ICASE, Hampton, Virginia

Institute for Computer Applications in Science and Engineering

NASA Langley Research Center

Hampton, VA

Operated by Universities Space Research Association

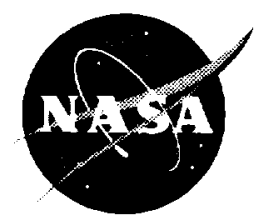

National Aeronautics and

Space Administration

Langley Research Center

Hampton, Virginia 23681-2199
Prepared for Langley Research Center under Contract NAS 1-97046

October 1999 
Available from the following:

NASA Center for AeroSpace Information (CASI)

7121 Standard Drive

Hanover. MD $21076-1.320$

(301) 621-0.390
National Technical Information Service (NTIS)

5285 Port Royal Road

Springfield. VA 22161-2171

(703) 487-4650 


\title{
CHARACTERIZATION OF SOUND RADIATION BY UNRESOLVED SCALES OF MOTION IN COMPUTATIONAL AEROACOUSTICS*
}

\author{
ROBERT RUBINSTEIN AND YE ZHOU
}

\begin{abstract}
Evaluation of the sound sources in a high Reynolds number turbulent flow requires timeaccurate resolution of an extremely large number of scales of motion. Direct numerical simulations will therefore remain infeasible for the forseeable future; although current large eddy simulation methods can resolve the largest scales of motion accurately, they must leave some scales of motion unresolved. A priori studies show that acoustic power can be underestimated significantly if the contribution of these unresolved scales is simply neglected.

In this paper, the problem of evaluating the sound radiation properties of the unresolved, subgrid-scale motions is approached in the spirit of the simplest subgrid stress models: the unresolved velocity field is treated as isotropic turbulence with statistical descriptors evaluated from the resolved field. The theory of isotropic turbulence is applied to derive formulas for the total power and the power spectral density of the sound radiated by a filtered velocity field. These quantities are compared with the corresponding quantities for the unfiltered field for a range of filter widths and Reynolds numbers.
\end{abstract}

Key words. aeroacoustics, subgrid sound, large eddy simulation, sound sources

Subject classification. Physical Sciences

1. Introduction. The evaluation of the sound sources in high Reynolds number turbulent flows presents a fundamental problem for computational aeroacoustics. At one extreme, if a turbulence transport model is used to compute single-point single-time moments of the turbulence, the uncertainty of the turbulence model is compounded by the uncertainty of modeling the two-point two-time statistics required to evaluate the sound source.

At the other extreme, direct numerical simulation (DNS) requires that a large number of scales of motion be resolved accurately in time. Typical estimates [11] are that the resolution requirements are of the order of $R e^{3}$. Since the sound source depends on time correlations, in principle the entire flow history must be stored; this would impose prohibitive storage requirements even to compute the sound radiated by a model flow like isotropic turbulence. Further difficulties are posed if the acoustic field is to be resolved by DNS as well [11].

A practical compromise appears to be emerging in which the sound sources are computed by LES and are propagated to the far field by an acoustic analogy $[10,5]$ or by solution of the linearized Euler equations $[4,2]$.

If the sound source is computed by large eddy simulation (LES), then the acoustic calculation will evaluate the sound radiated by the resolved velocity field alone. It can be anticipated that this will result at least in the suppression of high-frequency sound. A priori studies $[11,14,16]$ suggest that this type of numerical sound suppression can be significant and motivate the present theoretical study of the relationship between the sound radiated by the exact velocity field and the sound radiated by the filtered velocity field.

\footnotetext{
*Institute for Computer Applications in Science and Engineering (ICASE), NASA Langley Research Center, Hampton, VA 23681-2199. This research was supported by the National Aeronautics and Space Administration under NASA Contract No. NAS1-97046 while the authors were in residence at the Institute for Computer Applications in Science and Engineering (ICASE), NASA Langley Research Center, Hampton, VA 23681-2199.
} 
This problem is addressed in the spirit of the simplest ideas used in subgrid stress modeling. Namely, we invoke Kolmogorov's theory of the universality of the small scales of motion in turbulence [3] and assume that the unresolved scales can be modeled as isotropic turbulence with statistical descriptors computed from the resolved velocity field. Using the theory of the space-time properties of isotropic turbulence, we can construct models of the exact and filtered velocity fields and compare the sound radiated by both fields as a function of filter width.

A different approach to subgrid-scale sound radiation is proposed by [14]. The present work differs from this primarily in the emphasis on time correlation modeling.

The present analysis is closely related to another modeling method in CAA, the stochastic synthesis of the subgrid motions $[1,4]$. Like these methods, the present analysis depends on a model for the two-point two-time properties of the subgrid motions. Thus, our model (Eqs. (2.7) and (2.8) below), could be used as the statistical descriptor required to synthesize the subgrid sound sources.

Precise comparisons with existing a priori studies $[11,14,16]$ are hampered by the very low Reynolds numbers of the direct simulations; the present analysis is appropriate when a Kolmogorov inertial range exists. Comparisons based on the non-universal spectra obtained in low Reynolds number simulations is possible, but less tractable analytically. This issue is discussed later.

2. The exact and filtered sound source. Denote the exact fluctuating velocity field by $\mathbf{u}(\mathbf{x}, t)$, and the filtered field by $\overline{\mathbf{u}}(\mathbf{x}, t)$. Only the sharp Fourier cutoff filter will be treated explicitly here, for which the space Fourier transform of the resolved velocity field is

$$
\overline{\mathbf{u}}(\mathbf{k}, t)= \begin{cases}\mathbf{u}(\mathbf{k}, t) & \text { if } k \leq k_{L} \\ 0 & \text { if } k \geq k_{L}\end{cases}
$$

where

$$
k_{L}=\pi / \Delta
$$

and $\Delta$ equals the physical space filter size. Extension of the analysis to other filters would be straightforward.

Lighthill's [10] formula for the acoustic pressure fluctuations in the far-field is

$$
p(\mathbf{x}, t)=\frac{1}{4 \pi r^{2}} \frac{x_{i} x_{j}}{x^{3}} \int_{1} d \mathbf{y} \ddot{T}_{i j}\left(\mathbf{y}, t-\frac{|\mathbf{x}-\mathbf{y}|}{c}\right)
$$

where the form of the Lighthill tensor for sound radiation by subsonic flow

$$
T_{i j}(\mathbf{y}, t)=\rho u_{i}(\mathbf{y}, t) u_{j}(\mathbf{y}, t)
$$

is used. In Eqs. (2.3) and (2.4), I denotes the source region, $\rho$ is the mean density, $c$ is the speed of sound in the far-field, and the vector $\mathbf{x}$ connects the measurement point to some representative point in the source region.

The sound radiated by the filtered velocity field has the same form as Eq. (2.3), but with the Lighthill tensor computed from the resolved, rather than the exact velocity:

$$
\bar{T}_{i j}(\mathbf{y}, t)=\rho \bar{u}_{i}(\mathbf{y}, t) \bar{u}_{j}(\mathbf{y}, t)
$$

Kraichnan [7] gave the far-field acoustic power spectral density as

$$
p(\omega)=\pi\left(\omega^{4} / 2 c^{8}\right)\left\langle\left|n_{i} n_{j} T_{i j}(\omega \mathbf{n} / c, \omega)\right|^{2}\right\rangle
$$


where $\mathbf{n}$ is the unit vector in the direction of $\mathbf{x}, \omega$ is the frequency of the radiated sound, and $T_{i j}(\mathbf{k}, \omega)$ is the space-time Fourier transform of the fluctuating quantity $T_{i j}(\mathbf{x}, t) . T_{i j}(\mathbf{k}, \omega)$ can be written in terms of the space-time Fourier transform of the velocity field as

$$
T_{i j}(\mathbf{k}, \omega)=\rho \int \delta(\mathbf{k}-\mathbf{p}-\mathbf{q}) d \mathbf{p} d \mathbf{q} \int_{\infty}^{\infty} d \omega^{\prime} u_{i}\left(\mathbf{p}, \omega-\omega^{\prime}\right) u_{j}\left(\mathbf{q}, \omega^{\prime}\right)
$$

It is necessary to express the result of Eq. (2.6) in terms of statistics of the velocity field. To this end, replace $T_{i j}$ in $\mathrm{Eq}$. (2.6) by result in Eq. (2.7). Close the resulting fourth-order velocity moment by quasinormality, and use the correlation function for isotropic turbulence

$$
Q_{i j}(\mathbf{k}, \omega)=\frac{1}{4 \pi k^{2}} E(k) R(k, \omega)\left[\delta_{i j}-k_{i} k_{j} k^{-2}\right]
$$

where $E(k)$ is the energy spectrum and $R(k, \omega)$ is the time correlation function.

It can be shown [13] that Eq. (2.6) implies that sound is radiated only by interactions between incompressible modes nearly of the type $u_{i}(\mathbf{k}, t)$ and $u_{j}(-\mathbf{k}, t)$. This approximation treats the sound waves as infinitely long; equivalently, it ignores the so-called retarded time effect. Introducing this approximation. the result $[12]$ is

$$
p(\omega)=C \frac{\omega^{4}}{\Gamma^{5} c^{5}} \int_{0}^{\infty} d k E(k)^{2} k^{-3} \hat{R}(k, \omega)
$$

where $\hat{R}$ denotes the frequency convolution

$$
\hat{R}(k, \omega)=\int_{-\infty}^{\infty} d \omega^{\prime} R\left(k, \omega-\omega^{\prime}\right) R\left(k, \omega^{\prime}\right)
$$

3. Sound radiation by the filtered velocity field. To complete the calculation of the far-field acoustic power spectral density function using Eq. (2.9), models for the energy spectrum $E(k)$ and the time correlation function $R(k, \omega)$ are needed. Since these will describe the unresolved motions, it is necessary to assume that these motions can be described reasonably well by some universal properties; for this description, we will apply the Kolmogorov theory of the inertial range [3].

Our previous work [12] discusses the issue of time correlations in detail. It is concluded that sound radiation is determined by Eulerian time correlations, for which turbulence theory $[9,15,6]$ demonstrates the similarity form

$$
R(k, \omega)=R\left(\frac{\omega}{V k}\right)
$$

for inertial range scales, where $V$ is the $r m s$ of the fluctuating velocity. A similar formula is proposed by Bailly and Juvé [2], although this work treats the frequencies corresponding to each wavevector as random variables.

Replacing $\hat{R}$ by the similarity form corresponding to Eq. (3.1),

$$
p(\omega)=C \frac{\omega^{4}}{V c^{5}} \int_{0}^{\infty} d k E(k)^{2} k^{-3} \hat{R}\left(\frac{\omega}{V k}\right)
$$

Next, let $E(k)$ be the Kolmogorov spectrum

$$
E(k)=C_{K} \varepsilon^{2 / 3} k^{-5 / 3}
$$

In reality, this form only applies to a finite range of scales $k_{0}<k<k_{d}$, where $k_{0}$ is the inverse integral scale, and $k_{d}$ is proportional to the inverse Kolmogorov scale. But the subsequent calculation will show that 
the exact form of the spectrum in the region of large scales $k \leq k_{0}$ is not important for the calculation of subgrid sound. Similarly, the spectrum in the dissipation range with $k \geq k_{d}$ can be neglected because of its insignificant energy content. Substituting Eq. (3.3) in Eq. (3.2),

$$
p(\omega)=C \frac{\varepsilon^{4 / 3}}{c^{5}} \omega^{4} \int_{0}^{\infty} d k k^{-19 / 3} \hat{R}\left(\frac{\omega}{V k}\right)
$$

If the time correlation function $\hat{R}$ decays sufficiently rapidly at $\infty$, this integral is finite for $k$ near 0 ; consequently, as noted earlier, the precise form of $E(k)$ for small $k$ is not needed for this calculation. Regardless of the functional form of $\hat{R}$, power counting shows that for large $\omega$,

$$
p(\omega) \approx C \frac{V^{13 / 3} \varepsilon^{4 / 3}}{c^{5}} \omega^{-4 / 3}
$$

Next, consider the sound radiated by the filtered velocity field. Denote its power spectral density by $p_{L}(\omega)$ to indicate the dependence on the filter scale $k_{L}$. We noted in the previous section that ignoring the retarded time effect means that only mode pairs of the form $u_{i}( \pm \mathbf{k}, t)$ interact to radiate sound [13]. If so, the sound radiated by the filtered velocity field $\bar{u}_{i}(\mathbf{k}, t)$ is found from Eq. (3.4) by restricting the $k$ integration to the resolved scales:

$$
p_{L}(\omega)=C \frac{\omega^{4}}{V c^{5}} \int_{0}^{k_{L}} d k E(k)^{2} k^{-3} \hat{R}\left(\frac{\omega}{\Gamma k}\right)
$$

with the obvious analog of $E_{q}$. (3.6) for the Kolmogorov spectrum

$$
p_{L}(\omega)=C \frac{\varepsilon^{4 / 3}}{c^{5}} \omega^{4} \int_{0}^{k_{L}} d k k^{-19 / 3} \hat{R}\left(\frac{\omega}{V k}\right)
$$

Analytical results will require assuming a specific functional form for the time correlation function. We note three forms:

1. Kraichnan's [8] result

$$
R(x)=\frac{J_{1}(x)}{x}
$$

2. the Markovian approximation

$$
R(x)=\exp (-|x|)
$$

3. the Gaussian approximation

$$
R(x)=\exp \left(-x^{2}\right)
$$

where $x$ is the similarity variable

$$
x=\alpha \frac{\omega}{V k}
$$

The constant $\alpha$ should be chosen to match the second order Taylor coefficient of Eqs. (3.8)-(3.11) to the short-time expansion of the Navier-Stokes equations following the analysis of Kaneda [6].

The analytically most convenient form is the Gaussian of Eq. (3.10), which is tentatively adopted here in order to illustrate the derivation of a theory of subgrid-scale sound. Regardless of which of Eqs. (3.8)-(3.10) is used, the final result can be written in the form

$$
\bar{p}_{L}(\omega)=C \frac{\varepsilon^{4 / 3} V^{13 / 3}}{c^{5}} \omega^{-4 / 3} F\left(\frac{\omega}{V k_{L}}\right)
$$




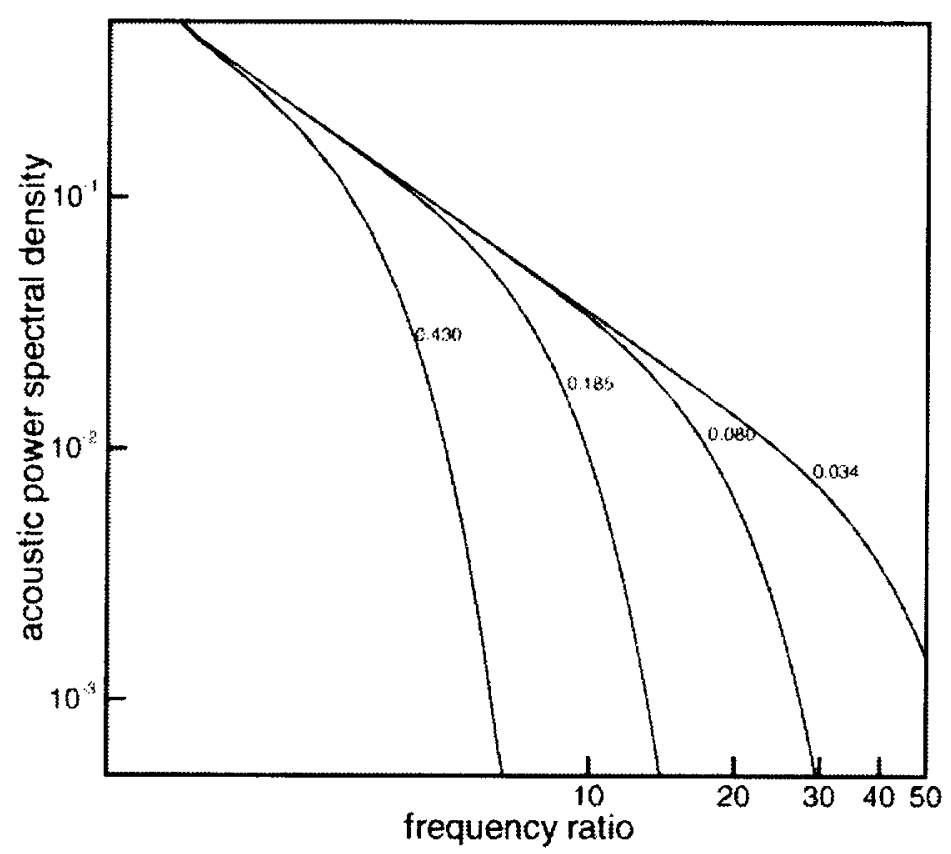

FIG. 3.1. Effect of filter size on acoustic power spectral density.

For Eq. (3.10), the function $F$ can be expressed as an incomplete gamma function.

The effect of filter width on the spectrum of sound radiated by a filtered velocity field is shown in Figure 3.1 in which the dimensionless power spectrum is plotted as a function of normalized frequency $\omega / \omega_{0}$ where $\omega_{0}$ is the frequency integral scale. The filter width is converted to a frequency $\omega_{L}$ through the relation

$$
\omega_{L}=V k_{L}
$$

and the spectrum in Eq. $(3.12)$ is plotted for the values $\omega_{0} / \omega_{L}=k_{0} / k_{L}=.9^{4} \approx 0.43, .9^{8} \approx 0.18, .9^{16} \approx$ $0.08, .9^{32} \approx 0.03$ ranging from extremely coarse to very fine resolution of the fluctuating field. The most conspicuous effect of coarsening the resolution is suppression of high frequency sound, although there is some effect at all frequencies.

In Figure 3.1, it is assumed that the inertial range extends over all scales and consequently that sound is radiated at all frequencies. But if it is assumed instead that the dissipation range spectrum is simply zero, then the acoustic spectrum would have the form of Eq. (3.12) with the filter scale $k_{L}$ replaced by the dissipation scale $k_{d}$. In this case, comparison between two curves in Figure 3.1 shows the effect of filtering a velocity field with a finite $k_{d}$.

Perhaps more immediately important is the effect of filter width on the computed total acoustic power. The exact total acoustic power is

$$
P=\int_{0}^{\infty} d \omega p(\omega)
$$

and the total power radiated by the resolved scales is

$$
P_{L}=\int_{0}^{\infty} d \omega p_{L}(\omega)
$$




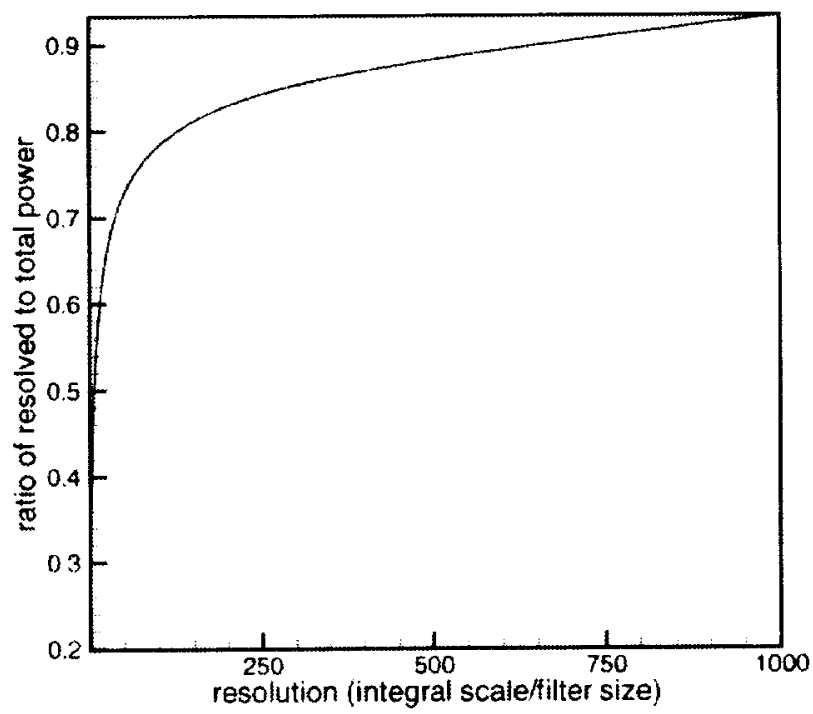

Fik. 3.2. Fiffert of filtering on total power.

Using $\mathrm{E}_{\mathrm{l}}$. (3.12), the ratio of resolved to total acoustic power is

$$
\frac{P_{L}}{P}=\frac{1}{3 F(0)} \int_{1}^{\left.\omega_{L} / \omega^{\prime}\right)} d \tilde{\omega}(\tilde{\omega})^{-4 / 3} F\left(\tilde{\omega} \frac{\omega_{0}}{\omega_{L}}\right)
$$

This function is shown in Figure 3.2 as a function of the variable $\omega_{L} / \omega_{0}=k_{L} / k_{0}$, the ratio of the integral scale to the filter size. Since the inertial range extends over all scales, even the largest value shown, which corresponds to a filter width of 0.001 times the integral scale, is only within about $1 \mathrm{~dB}$ of the total power. For filter widths less than 0.01 times the integral scale, the resolved power rises rapidly: at 0.01 times the integral scale, the resolved power is within about $3 \mathrm{~dB}$ of the total.

As before, the ratio $P_{L} / P_{d}$ where $P_{d}$ is the power in a Kolmogorov spectrum cut off sharply at scale $k_{d}$, is a good approximation for the ratio of resolved to total power for a finite Reynolds number flow. Figure 3.2 therefore provides a single curve showing the combined effects of Reynolds number and resolution.

By setting $k_{d} / k_{0}=100$, we obtain a Reynolds number of about 460 . The ratio of resolved to theoretical total power $P_{L} / P_{d}$ is plotted for this case in Figure 3.3, together with the same ratio in the infinite Reynolds number limit. The "resolution" is the ratio of the integral scale to the filter size: although it might be more natural to define resolution as the ratio of the filter size to the Kolmogorov scale, the results for different Reynolds numbers could not be compared directly since the Kolmogorov scales corresponding to the two different Reynolds numbers would be unequal. As expected, the unresolved power in the infinite Reynolds number limit always exceeds the unresolved power in the finite Reynolds number flow. In both cases, the subgrid power drops sharply as the resolution is increased over low values, but tends to drop slowly at higher resolution.

All of these estimates of the acoustic power in the subgrid scales must be considered preliminary because of the assumption of the Gaussian time correlation function which has been chosen largely for analytical convenience. 


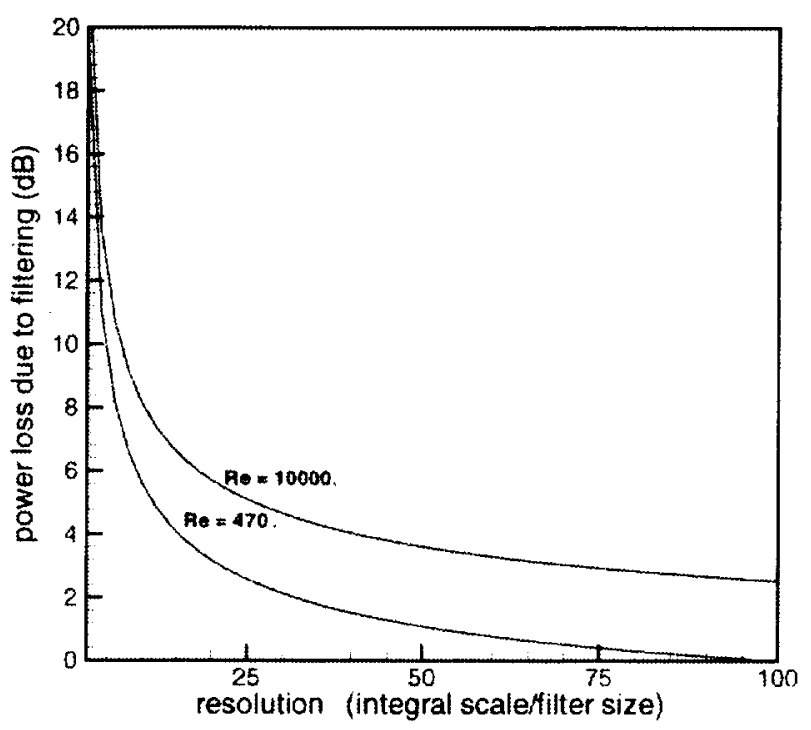

Fı(i. 3.3. Power loss due to filtering: Effect of Reynolds number.

The problem of acoustic power radiated by subgrid scales has been addressed in a priori studies by Piomelli et al. [11] for the model problem of channel flow and by Witkowska et al. [16] for the problem considered here of isotropic turbulence. The latter study considers both forcod. steady turbulence and the more commonly studied problem of decaying turbulence. Comparison with these results requires some care because of the limited Reynolds numbers of the simulations. This comparison must be based on results like Eq. (3.6) expressed in terms of a numerically given spectrum $E(k)$ instead of the holnogorov spectrum used here.

4. Conclusions. The present work is a first step to an analytical theory of the subgricl contribution to radiated sound. It shows how the theory of isotropic turbulence can be applied to derive a theory of subgridscale sound radiation. Refinement of this model will require closer investigation of the time correlation function, which is the key ingredient of our analysis.

The close connection of this work to the method of stochastic synthesis advanced by Baily et al. $[1,2]$ and by Béchara et al. [4] was noted earlier. Although this application has not been developed explicitly here, the present theory is based on a two-point two-time model of the subgrid scales which could also be used to synthesize the subgrid-scale motions.

\section{REFERENCES}

[1] C. Bailly, P. Lafon, and S. Candel, Subsonic and supersonic jet noise predictions from statistical source models, AIAA J. 35 (1997), p. 688.

[2] C. BAIlly AND D. JuvÉ, A stochastic approach to compute subsonic noise using linearized Euler equations, AIAA Paper 99-1872, 1999.

[3] G.K. Batchelor, The theory of homogeneous turbulence, Cambridge University Press, 1948. 
[4] W. BÉChara, P. Lafon, And C. Bailly, Applications of a k- $\epsilon$ turbulence model to the prediction of noise for simple and coaxial free jets, J. Acoust. Soc. Am. 97 (1995), p. 3518.

[5] J.E. FFowCS-WILliams and D.L. Hawkincis, Sound generation by turbulence and surfaces in arbitrary motion, Phil. Trans. 264A (1969), p. 321.

[6] Y. KANEdA, Lagrangian and Eulerian time correlations in turbulence, Phys. Fluids A 5 (1993), p. 2835.

[7] R.H. Kratchnan, The scattering of sound in a turbulent medium, J. Acoust. Soc. Am. 25 (1953), p. 1093.

[8] R.H. KRaichnan, The structure of isotropic turbulence at very high Reynolds number, J. Fluid Mech. 5 (1959), p. 497.

[9] R.H. Kraichnan, Kolmogorov's hypotheses and Eulerian turbulence theory, Phys. Fluids 7 (1964), p. 1723 .

[10] M.J. Lighthill, On sound generated aerodynamically: I. General theory, Proc. Roy. Soc. A211 (1952), p. 1107 .

[11] U. Piomelli, C.L. STreett, and S. Sarkar, On the computation of sound by large-eddy simulations, J. Eng. Math. 32 (1997), p. 217.

[12] R. Rubinstein And Y. ZhOt, Time correlations and the frequency spectrum of sound radiated by turbulent flows, ICASE Report 97-7, 1997.

[13] R. Rubinstein ano Y. Zhol; Effects of helicity on Lagrangian and Eulerian time correlations in turbulence. Phys. Fluids 11 (1999), p. 2288.

[14] C. Seror, P. Sagaut, C. Ballly, and D. Juvé, Subgrid scale contribution to noise production in decaying isotropic turbulence, AIAA Paper 99-1979, 1999.

[15] H. Tennekes, Eulerian and Lagrangian time microscales in isotropic turbulence, J. Fluid Mech. 67 $(1975)$, p. 561.

[16] A. Witkowska, D. Juvé, AND J.G. Brasselk, Numerical study of noise from isotropic turbulence, J. Comput. Acoust. 5 (1997), p. 317.

[17] Y. ZhOl:, And R. RUbinstein, Sweeping and straining effects in sound generation by high Reynolds number isotropic turbulence, Phys. Fluids 8 (1996), p. 647. 



\section{REPORT DOCUMENTATION PAGE}

Public reporting burden for this collection of information is estimated to average I hour per response, including the time for reviewing instructions, searching existing data sources. gathering and maintaining the data needed, and completing and reviewing the collection of information. Send comments regarding this burden estimate or any other aspect of this Davis Highway, Suite 1204, Artington, VA 22202-4302, and to the Office of Management and Budget. Paperwork Reduction Project (0704-0188), Washington, DC 20503.

\begin{tabular}{|l|c|c|}
\hline 1. AGENCY USE ONLY(Leave blank) & $\begin{array}{c}\text { 2. REPORT DATE } \\
\text { October } 1999\end{array}$ & $\begin{array}{l}\text { 3. REPORT TYPE AND DATES COVERED } \\
\text { Contractor Report }\end{array}$
\end{tabular}

\section{TITLE AND SUBTITLE}

5. FUNDING NUMBERS

Claracterization of sound radiated by unresolved scales of motion in computational aeroacoustics

C NAS1-97046

WU 505-90-52-01

6. AUTHOR(S)

Robert Rubinstein

Ie: Zliou

7. PERFORMING ORGANIZATION NAME(S) AND ADDRESS(ES)

Institute for Computer Applications in Science and Engineering

Mail Stop 132C, NASA Langley Research Center

Hampton, VA 23681-2199

9. SPONSORING/MONITORING AGENCY NAME(5) AND ADDRESS(ES)

National Aeronautics and Space Administration

Langley Research Center

Hampton, VA 23681-2199
8. PERFORMING ORGANIZATION REPORT NUMBER

ICASE Report No. 99-39

\section{SUPPLEMENTARY NOTES}

Langley Technical Monitor: Denuis M. Bushnell

Final Report

Submitted to the European Journal of Mechanics B.

12a. DISTRIBUTION/AVAILABILITY STATEMENT

12b. DISTRIBUTION COOE

Unclassified - Unlinited

Subject Category 34

Distribution: Nonstandard

Availability: NASA-CASI (301) 621-0390

13. ABSTRACT (Maximum 200 words)

Evaluation of the sound sources in a high Reynolds number turbulent flow requires time-accurate resolution of an extremely large number of scales of motion. Direct numerical simulations will therefore remain infeasible for the forseeable future: althongh current large eddy simulation methods can resolve the largest scales of motion arcurately, they must leave some scales of motion unresolved. A priori studies show that acoustic power can be underestimated signiticantly if the contribution of these unresolved scales is simply neglected.

In this paper, the problem of evaluating the sound radiation properties of the unresolved, subgrid-scale motions is approached in the spirit of the simplest subgrid stress models: the unresolved velocity field is treated as isotropic turbulence with statistical descriptors evaluated from the resolved field. The theory of isotropic turbulence is applied to derive formulas for the total power and the power spectral density of the sound radiated by a filtered velocity field. These quantities are compared with the corresponding quantities for the unfiltered field for a range of filter widtlss and Revmolds numbers.

14. SUBJECT TERMS

aeroacoustics, subgrid sound, large eddy simulation, sound sources

15. NUMBER OF PAGES 13

16. PRICE CODE A03

\begin{tabular}{l|l|l|}
\hline 17. SECURITY CLASSIFICATION & $\begin{array}{l}\text { 18. SECURITY CLASSIFICATION } \\
\text { OF REPORT } \\
\text { OF THIS PAGE } \\
\text { Unclassified }\end{array}$ & $\begin{array}{l}\text { 19. SECURITY CLASSIFICATION } \\
\text { OF ABSTRACT }\end{array}$ \\
\hline
\end{tabular}

20. LIMITATION OF ABSTRACT 